\title{
La normalización de la descripción archivística en Gran Bretaña, EE.UU., Canadá, Portugal, Brasil y España después de ISAD(g): procesos y modelos de trabajo
}

\author{
Dunia LLanes Padrón \\ duniallp@yahoo.es \\ Universidad de La Habana
}

\begin{abstract}
Resumo: Se estudian y contextualizan los procesos normativos desarrollados en los países que han publicado estándares después de la edición de ISAD(G): Gran Bretaña, EE.UU, Canadá, Portugal, Brasil y España (tanto a nivel nacional como en las comunidades de Castilla y León, Cataluña, Galicia y Aragón). En este sentido, se realiza una reflexión sobre la perspectiva histórica de la descripción en estas naciones y comunidades autonómicas, sistematizando las actividades realizadas hasta la publicación oficial de los estándares.
\end{abstract}

Palavras-chave: descripción archivística; representación de documentos; normas de descripción; procesos normativos de descripción.

Abstract: The policy processes developed in some countries that have published regulatory texts, after the edition of $\operatorname{ISAD}(G)$ : Great Britain, USA, Canada, Portugal, Brazil and Spain (both nationally and in communities of Castile and Leon, Cataluña, Galicia and Aragon) are, also, studied and contextualized. In this sense, there is a reflection on the historical perspective of description in these nations and autonomous regions, systematizing the activities which took place until the official standards' publication.

Keywords: archival description; documents representation; descriptive standards; standardized description processes.

\section{INTRODUCCIÓN}

La década de 1990 trajo consigo varios cambios en los paradigmas de la descripción archivística. La actividad de describir fue modificada como consecuencia de las nuevas tecnologías y del proceso internacional de normalización. La publicación de las normas ISAD(G), ISAAR(CPF), ISDF e ISIAH fueron de los resultados de carácter internacional más importantes de esta área. El surgimiento de estos estándares puso de manifiesto la posibilidad de elaborar proyectos normativos que tomaran como punto de partida los proyectos internacionales.

En este sentido, en la segunda edición de la $\operatorname{ISAD}(G)$ se encontraba una recomendación precisa, según la cual "esta norma constituye una guía general para la elaboración de 
descripciones archivisticas" por lo que "debe utilizarse conjuntamente con las normas nacionales existentes o como base para el desarrollo de otras normas nacionales" (CONSEJO..., 2000, p.12).

Atendiendo a esta sugerencia, diferentes países y comunidades autónomas prepararon y publicaron normas para la descripción archivística, adaptándolas a sus realidades teóricas y prácticas. Gran Bretaña, EE.UU, Canadá, Portugal, Brasil y España son los países que han desarrollado nuevos estándares descriptivos. Los archiveros de estas naciones buscaban alcanzar modelos que se ajustaran a las tradiciones archivísticas y que sistematizaran los procedimientos e instrumentos de descripción existentes.

Resulta imprescindible conocer los procesos normativos nacionales y regionales realizados por estos países así como las actividades desarrolladas pues constituyen un antecedente importante para la creación de nuevas normas nacionales. Esta investigación tiene como objetivo describir los métodos de trabajo seguidos por estos países para crear estándares de descripción, con el fin de definir una guía de actividades comunes a cualquier proceso normativo.

A continuación se analizan y presentan por separado los procesos en cada uno de los países mencionados.

\section{EL PROCESO DE NORMALIZACIÓN EN GRAN BRETAÑA: MAD3 Y RCPPCN}

Uno de los pasos más significativos de Gran Bretaña en el campo de la descripción archivística fue la publicación de las dos ediciones del Manual of Archival Description (MAD1 y MAD2) entre los años 1986 y 1990. Según el profesor Michael Cook de la Universidad de Liverpool, Gran Bretaña fue el país representante de la tradición archivística europea frente a la americana en el ámbito de la descripción archivística, antes del surgimiento de ISAD(G).

MAD2 fue una de las normas nacionales que más aportó al proceso internacional de descripción (estructura y principios teóricos). No obstante, en Gran Bretaña, el desarrollo normativo se vio afectado, en algunos años, por varios factores relacionados con el escaso progreso de una base teórica en el campo de la archivística, el aislamiento y los esfuerzos individuales de los profesionales $y$, por último, el encuentro entre los defensores de una archivística beneficiada por las nuevas tecnologías y los defensores de una archivística tradicional.

En la actualidad, los archiveros de Gran Bretaña cuentan con dos normas que buscan la normalización de las prácticas descriptivas en los archivos: las Rules for the Construction of Personal, Place and Corporate Names (RCPPCN) para la normalización de los puntos de acceso 
y la tercera edición del Manual of Archival Description (MAD3) para la descripción de diferentes entidades y subentidades archivísticas. Resulta de interés conocer cuáles fueron las actividades que se desarrollaron en Gran Bretaña para el desarrollo de estas dos normativas que rigen, hoy, el proceso de descripción en este país. A continuación se listan las actividades desarrolladas para crear la Rules for the Construction of Personal, Place and Corporate Names y el Manual of Archival Description.

Tabla 1. Cronología de actividades realizadas en Gran Bretaña para la publicación de los dos estándares.

\begin{tabular}{|c|c|}
\hline \multicolumn{2}{|r|}{ RULES FOR THE CONSTRUCTION OF PERSONAL, PLACE AND CORPORATE NAMES } \\
\hline FECHAS & ACTIVIDADES \\
\hline 1990 & $\begin{array}{l}\text { - El National Council of Archives (NCA) constituyó un primer grupo de normalización para dar } \\
\text { comienzo a la creación de un registro de autoridades. }\end{array}$ \\
\hline 1993 & $\begin{array}{l}\text { - Se nombró, finalmente, un comité permanente que sería el responsable de preparar y redactar } \\
\text { las reglas para la formación del fichero de autoridades de nombres. Este tendría la } \\
\text { responsabilidad de unificar las reglas de catalogación de las instituciones británicas y resolver los } \\
\text { problemas prácticos de descripción de estas instituciones. } \\
\text { - Este grupo estuvo formado por integrantes de las siguientes instituciones: Historical Manuscripts } \\
\text { Commission, Public Record Office, British Library, National Library of Wales, Public Record Office of } \\
\text { Northern Ireland, Scottish Record Office, Victoria and Albert Museum, National Art Library. }\end{array}$ \\
\hline 1994 & $\begin{array}{l}\text { - El comité permanente de normalización estableció la creación de equipos que trabajaran por } \\
\text { separado en la redacción de reglas para los nombres de personas, lugares e instituciones. } \\
\text { - Se acordó que una vez elaborada la propuesta se presentaría al National Council of Archives para } \\
\text { su aprobación y, posteriormente, se enviaría a la comunidad profesional para conocer sus } \\
\text { comentarios. }\end{array}$ \\
\hline 1990-1996 & $\begin{array}{l}\text { - Trabajos de conjunto para la formación de reglas generales y específica, acompañadas de } \\
\text { ejemplos, para la creación normalizada de nombres de personas, familias e instituciones } \\
\text { vinculadas con la creación de documentos archivísticos. }\end{array}$ \\
\hline 1996 & $\begin{array}{l}\text { - Se obtiene el primer borrador de las reglas. } \\
\text { - Se difundió el borrador de las reglas en la comunidad archivística británica. } \\
\text { - Se recibieron opiniones tanto de instituciones como de archiveros individuales que ayudaron a } \\
\text { mejorar el borrador. }\end{array}$ \\
\hline 1997 & $\begin{array}{l}\text { Se publica la versión final y oficial de las Rules for the Construction of Personal, Place and } \\
\text { Corporate Names. }\end{array}$ \\
\hline \multicolumn{2}{|r|}{ MANUAL OF ARCHIVAL DESCRIPTION } \\
\hline FECHAS & ACTIVIDADES \\
\hline 1998 & $\begin{array}{l}\text { - Se decide realizar una tercera edición del Manual of Archival Description. } \\
\text { - La tercera edición de MAD fue organizada, redactada y dirigida por los profesores Margaret } \\
\text { Procter y Michael Cook, ambos de la Universidad de Liverpool. }\end{array}$ \\
\hline $\begin{array}{c}1998- \\
2000\end{array}$ & $\begin{array}{l}\text { - Se realizaron un conjunto de revisiones y cambios en la segunda edición de MAD. } \\
\text {-Los autores contaron con el apoyo de varias universidades y archivos ingleses, la Society of } \\
\text { American Archivists y el Consejo Internacional de Archivos (CIA). }\end{array}$ \\
\hline 2000 & - Se publica, oficialmente, la tercera edición del Manual of Archival Description. \\
\hline
\end{tabular}

Fuente: Elaborado por la autora.

Los británicos organizaron todo el trabajo para la estandarización de las autoridades bajo el auspicio y dirección del National Council of Archives. Crearon un comité de 
normalización donde participaron representantes de todas las instituciones de información con reputación del Reino Unido. Seguidamente, formaron grupos individuales para elaborar cada una de las reglas (nombres de personas, familias, instituciones y lugares). Establecieron un pequeño grupo editorial para la redacción del borrador y la versión oficial. Finalmente, involucraron a toda la comunidad archivística para conocer sus opiniones y tomarlas en consideración en la versión final de las Rules for the Construction of Personal, Place and Corporate Names.

Las Rules for the Construction of Personal, Place and Corporate Names constituyen la normativa que sirve de guía a todas las instituciones de información británicas para la creación de los puntos de acceso y los registros de autoridad. Estas reglas tienen como objeto ayudar a los archiveros a la formación de lenguajes documentales controlados que faciliten la búsqueda y la recuperación de la información en los archivos.

Por su parte, la tercera edición del Manual of Archival Description fue realizada por dos prestigiosos profesionales del área y tuvo gran aceptación dentro de la comunidad de archiveros ingleses. Esta obra sirve de libro de texto en las universidades que tienen dentro de sus programas el curso de archivo y, además, se utiliza como guía para la elaboración de los instrumentos de descripción.

\section{EL PROCESO DE NORMALIZACIÓN EN EE.UU: DACS}

La comunidad de bibliotecarios y archiveros de EE.UU ha estado inmersa, desde el siglo $\mathrm{XIX}$, en un constante proceso para facilitar a los usuarios el acceso a las fuentes de información. Este país es considerado pionero en las prácticas de normalización de la descripción archivística. Desde 1983 contaba con la norma de contenido Archives, Personal Papers and Manuscripts (APPM) y una norma de formato para el intercambio de información archivística United States Machinal Readable Cataloging for Archives and Manuscripts Control (USMARC AMC).

El desarrollo normativo de EE.UU. en la década de los 80 marcó pautas y tendencias que, posteriormente, constituyeron la base, junto a proyectos de otros países, de las normas internacionales.

Con la publicación de las normas del Consejo Internacional de Archivos (CIA) en la década de los 90, los profesionales vinculados a las ciencias de la información en EE.UU comenzaron a dar los primeros pasos para la formación de nuevas normas de estructura, contenido y formato, tratando de mantener la compatibilidad con los estándares internacionales. Es en este contexto cuando se inician los trabajos en del proyecto Canadian- 
US Task Force on Archival Description (CUSTARD) y, posteriormente, los de Describing Archives: a Content Standard (DACS), Encoded Archival Description (EAD), y Encoded Archival Context (EAC). Todos estos trabajos constituyen la base normativa de la descripción archivística en este país.

No es posible hablar de normalización en EE.UU., en la última década, sin reflexionar, previamente, sobre el trabajo realizado en conjunto con Canadá en el llamado proyecto CUSTARD.

En 1996 un grupo formado por archiveros americanos y canadienses, representantes de la Society of American Archivists y del Bureau of Canadian Archivists, decidieron trabajar en conjunto para desarrollar una norma norteamericana, motivados por la proliferación de la normalización de la descripción archivística a nivel internacional. Este proyecto es conocido por el nombre de CUSTARD.

El objetivo principal del proyecto fue crear una nueva norma que recogiera todos los elementos de ISAD(G) e ISAAR(CPF) y reemplazara a APPM y a las reglas canadienses de descripción; y que se convirtiera en una nueva norma de contenido que se ajustara a las normas estructurales de descripción existentes.

Durante varios años, los archiveros, de ambos países, trabajaron en conjunto y lograron obtener los primeros borradores de lo que sería la norma norteamericana. También se precisaron los principios del proyecto, que se basaron en la teoría archivística de la descripción y en las reglas establecidas por $\operatorname{ISAD}(G)$. Como consecuencia de esto surgió la Declaración de Principios del Proyecto CUSTARD. Esta declaración, después, fue utilizada como base para el desarrollo de otros estándares nacionales.

Los integrantes del proyecto CUSTARD decidieron que la nueva norma que llevaría por nombre Describing Archives: A Content Standard reflejaría una aproximación más amplia de la descripción archivística que APPM y serviría de contenido de datos para EAD.

Finalmente, el proyecto tomó un curso distinto por las diferencias teóricas y conceptuales irreconciliables entre los miembros estadounidenses y canadienses del comité. Los archiveros estadounidenses plantearon no estar de acuerdo con incluir las reglas que proponían los canadienses para describir todo tipo de documento, opinaban que iban a existir demasiadas semejanzas con las Anglo American Cataloguing Rules.

Por su parte, los archiveros canadienses decidieron no aceptar DACS como la norma canadiense. Todo parece indicar que el Bureau of Canadian Archivists no aceptó la perspectiva, fuertemente, custodial del proyecto CUSTARD porque rompía con su tradición de archivos totales y, por lo tanto, se apartaron del proyecto. 
En tanto, los archiveros americanos completaron la revisión final, la edición y publicación de DACS. La Society of American Archivists aprobó oficialmente DACS, en el 2004, como norma nacional de contenido de datos y como sustituta de APPM.

El proyecto CUSTARD fue una iniciativa válida para crear un proyecto descriptivo común ejecutable en los archivos norteamericanos. Durante años de trabajo conjunto se desarrollaron varias actividades que en el futuro fueron aprovechadas en beneficio de ambos países. Este intento sirvió de ejemplo a otros países que se basaron en esta experiencia y en la declaración de principios del proyecto.

A continuación se describen las principales actividades desarrolladas hasta la publicación de DACS, incluyendo las actividades de CUSTARD:

Tabla 2. Actividades desarrolladas por el grupo CUSTARD

\begin{tabular}{|c|c|}
\hline \multicolumn{2}{|r|}{ DESCRIBING ARCHIVES: A CONTENT STANDARD } \\
\hline FECHAS & ACTIVIDADES \\
\hline 1996 & $\begin{array}{l}\text { - Se crea el Canadá-US. TaskForce on Archival Description. Este comité estuvo integrado por } \\
\text { profesionales de EE.UU y Canadá que anteriormente estuvieron vinculados a proyectos } \\
\text { normativos internacionales, nacionales e institucionales. }\end{array}$ \\
\hline 1999 & $\begin{array}{l}\text { - Se reúne el grupo en Toronto y elabora el "Toronto Accord for Descriptive Standards". Este } \\
\text { acuerdo recoge los principales objetivos de este grupo y planteó la necesidad de revisión de la } \\
\text { terminología para compatibilizar los estilos y concepciones de ambos países }\end{array}$ \\
\hline 2001 & $\begin{array}{l}\text { - La Sociedad de Archiveros Americanos recibió una beca de la National Endowment for } \\
\text { Humanities para financiar el trabajo del proyecto CUSTARD. } \\
\text { - Se comenzaron a elaborar los primeros borradores de la norma, redactados y revisados por el } \\
\text { grupo de archiveros que integraban el proyecto. } \\
\text { - Se elabora la Declaración de Principios del Proyecto CUSTARD. }\end{array}$ \\
\hline 2002-2003 & $\begin{array}{l}\text { - Se realizaron intercambios presenciales entre los miembros de CUSTARD y, también, } \\
\text { intercambios de opiniones a través del correo electrónico. } \\
\text { - Se presentó el proyecto a la comunidad de archiveros norteamericanos para que pudieran dar } \\
\text { sus opiniones sobre la norma. Se pretendía que la publicación de la norma tuviera una } \\
\text { aceptación total en la comunidad de archiveros norteamericana. }\end{array}$ \\
\hline 2003 & $\begin{array}{l}\text { Se rompe el proyecto CUSTARD por diferencias teóricas y prácticas entre archiveros } \\
\text { americanos y canadienses. }\end{array}$ \\
\hline 2004 & $\begin{array}{l}\text { - La Society of American Archivists publica, oficialmente, Describing Archives: a Content } \\
\text { Standard como la norma de descripción de EE.UU. }\end{array}$ \\
\hline
\end{tabular}

Fuente: Elaborado por la autora.

En la actualidad DACS es una norma exhaustiva y global. Es considerada un estándar de estructura, contenido y codificación y a través de ella se pueden describir documentos, productores y crear puntos de acceso normalizados. Los archiveros americanos la adoptaron inmediatamente y comenzaron a asistir a talleres de trabajo para poner en práctica la norma. 
El modelo de trabajo utilizado en la confección de DACS tuvo un gran inconveniente: involucrar a países diferentes en el desarrollo de una norma archivística común. El fracaso del proyecto americano y canadiense demostró la dificultad que entraña unificar regiones para elaborar y consolidar normas descriptivas archivísticas. Las diferencias teóricas, conceptuales y prácticas entorpecen el éxito en este tipo de trabajo.

\section{EL PROCESO DE NORMALIZACIÓN EN CANADÁ: RAD2}

La archivística canadiense ha estado en un proceso de desarrollo continuo desde la década de los 80. La normalización de la descripción es uno de los resultados de los avances teóricos y normativos desarrollados por las asociaciones profesionales de este país. El desarrollo de la normalización de la descripción en Canadá se llevó a cabo en varias etapas comprendidas entre 1978 y 1996. En 1990 los archiveros canadienses publicaron los primeros capítulos de las Rules for Archival Description (RAD).

En 1996 después de haber transcurrido algunos años de la publicación de ISAD(G), la comunidad archivística canadiense completó la publicación de sus regalas generales RAD en francés y en inglés. Wendy Duff señalaba que "estas reglas pretendían apoyar la creación de todo tipo de instrumento de descripción para todo tipo de material informativo y reflejarían la aproximación canadiense a los archivos totales" (DUFF, 2008, p.56). Los archivistas canadienses perseguían construir reglas para cada tipo de documento.

Como se indicó con anterioridad, los archiveros canadienses se unieron a los archiveros americanos en el proyecto CUSTARD, con el fin de crear un estándar normativo para Norteamérica. Después del mencionado fracaso por las respectivas divergencias entre los dos países, el comité responsable de la normalización en Canadá acordó utilizar la versión final de DACS con algunas revisiones para crear RAD2.

Para visualizar mejor el proceso de normalización en Canadá, a continuación se listan las actividades más importantes realizadas para publicar las RAD2. 
Tabla 3. Actividades realizadas para la publicación de RAD2

\begin{tabular}{|c|c|}
\hline \multicolumn{2}{|r|}{ RULES FOR ARCHIVAL DESCRIPTION 2} \\
\hline FECHA & ACTIVIDADES \\
\hline 1990 & $\begin{array}{l}\text { - Antes de la publicación de ISAD(G), los archiveros canadienses publican los primeros capítulos } \\
\text { de las Rules Archival Description. }\end{array}$ \\
\hline 1996 & $\begin{array}{l}\text { - Se completó la publicación de la primera edición completa de las Rules Archival Description en } \\
\text { francés y en inglés. }\end{array}$ \\
\hline 1996 & $\begin{array}{l}\text { - Se estableció el Canadian Committee on Archival Description (CCAD) que se responsabilizó del } \\
\text { mantenimiento, interpretación y revisión de la primera edición de RAD y fue el responsable de } \\
\text { la publicación de las RAD2. } \\
\text { EI CCAD estuvo formado por integrantes de la Association des Archivistes du Québec, la } \\
\text { Association of Canadien Archivists, National Archives of Canadá y la Library and Archives } \\
\text { Canadá. }\end{array}$ \\
\hline 1996 & $\begin{array}{l}\text { - Se crearon de grupos de trabajos independientes para redactar las reglas específicas de los } \\
\text { distintos tipos documentales (documentos sonoros, gráficos, fílmicos, textuales y electrónicos, } \\
\text { mapas, fotografías, dibujos, documentos filatélicos y objetos). Además de dos equipos que se } \\
\text { especializaron en la descripción del nivel fondo y la creación de puntos de acceso. }\end{array}$ \\
\hline 2001 & $\begin{array}{l}\text { - Los archivistas canadienses se integran, definitivamente, al proyecto CUSTARD y comienzan a } \\
\text { trabajar en los borradores para una norma norteamericana común. }\end{array}$ \\
\hline 2003 & $\begin{array}{l}\text { - Ruptura del proyecto CUSTARD. } \\
\text { - El comité canadiense decide utilizar el borrador de DACS con algunas revisiones y la primera } \\
\text { versión de RAD para publicar la segunda edición de las reglas canadienses. }\end{array}$ \\
\hline 2004 & $\begin{array}{l}\text { - Se difunde el primer borrador de RAD2. } \\
\text { - La comunidad archivística no reaccionó positivamente ante la propuesta del borrador de } \\
\text { RAD2. }\end{array}$ \\
\hline 2006 & $\begin{array}{l}\text { - El comité de elaboración solicitó más comentarios a la comunidad archivista. } \\
\text { - Se redactó un informe que se llamó: Hacia una segunda edición de RAD. }\end{array}$ \\
\hline 2008 & Se publica RAD2 como norma oficial para la descripción archivística en Canadá. \\
\hline
\end{tabular}

Fuente: Elaborado por la autora.

La publicación y revisión de RAD2, en 2008, fue el resultado del esfuerzo y la responsabilidad del comité de elaboración de la norma que respondió, eficazmente, a las necesidades de los archiveros canadienses. Esta revisión incluyó nuevas reglas de contenido y nuevos capítulos relacionados con los documentos electrónicos.

RAD2 es consecuencia del desarrollo archivístico canadiense y del alto grado de estandarización que ha tenido este campo en Canadá. Los cambios introducidos en la revisión de RAD permiten a las instituciones conseguir más flexibilidad en las descripciones y un desarrollo de esta área fundamentada en los principios de la práctica descriptiva contemporánea. 


\section{EL PROCESO DE NORMALIZACIÓN EN PORTUGAL: ODA}

El esfuerzo por coordinar una política nacional en los archivos portugueses es bastante reciente, data del año 1992 con la creación del Instituto dos Arquivos Nacionais /Torre do Tombo (IAN/TT). En cuanto a la normalización de la descripción, antes de la creación de este instituto, existía una diversidad de prácticas archivísticas basadas en instrumentos de trabajo y normas propias de cada archivo. Estos instrumentos de descripción constituían esfuerzos aislados y no estaban en consonancia unos con otros.

No es hasta el surgimiento de $\operatorname{ISAD(G)}$ que se puede hablar de un proceso de normalización de la descripción archivística en Portugal. A partir de 1996 el IAN/TT comienza a trabajar en proyectos y líneas de investigación vinculadas a la descripción. Las normas internacionales habían supuesto un desafío para esta área en el contexto portugués y nuevas exigencias para los profesionales.

El IAN/TT empieza un estudio pormenorizado de las dos normas internacionales. Como resultado de su análisis y aplicación surgieron varios documentos. Estos trabajos sirvieron de base para la futura conformación de una norma nacional. Recogieron los principios teóricos para la descripción de las entidades y la recuperación de la información.

Por la envergadura de este tema, el IAN/TT comprendió la necesidad de crear un comité que se dedicara, sólo, a la normalización y a la elaboración de una normativa de descripción archivística para el país. El grupo, designado como responsable de esta actividad, se propuso desarrollar unas orientaciones de descripción nacionales que fueran asumidas junto a la normativa internacional como normas generales.

A continuación se muestran las actividades realizadas en Portugal para la elaboración de estas orientaciones.

Tabla 4. Actividades realizadas para la publicación de ODA

\begin{tabular}{|c|c|}
\hline \multicolumn{2}{|r|}{ ORIENTAÇÕES PARA A DESCRIÇÃO ARQUIVÍSTICA } \\
\hline FECHAS & ACTIVIDADES \\
\hline 1996 & $\begin{array}{l}\text { - El Instituto de los Archivos Nacionales/Torre de Tombo comienza las actividades de } \\
\text { normalización de la descripción. } \\
\text { - Se realiza un estudio pormenorizado de las dos normas internacionales ISAD(G) e ISAAR(CPF). }\end{array}$ \\
\hline 1999 & - Realización del "Proyecto de Reglas Portuguesas de Descripción Archivistica”. \\
\hline 2000 & $\begin{array}{l}\text { - Elaboración del Proyecto de Reglas para la Construcción de un Registro Nacional de } \\
\text { Autoridades. }\end{array}$ \\
\hline 2000-2003 & $\begin{array}{l}\text { - Se crea el Grupo de Trabalho de Normalização da Descrição em Arquivo. En este grupo } \\
\text { estuvieron representadas todas las instituciones del país vinculadas a la archivística y a las } \\
\text { ciencias de la información en general. } \\
\text {-Se redacta el Programa de Normalización de la Descripción en Archivo. Este documento } \\
\text { estableció los pasos a seguir para la formación de la norma nacional. }\end{array}$ \\
\hline 2004 & - Traducción al portugués de la norma ISAD(G) y la ISAAR(CPF). \\
\hline
\end{tabular}




\begin{tabular}{|c|l|}
\hline 2006 & $\begin{array}{l}\text { - Redacción de la primera parte de la Norma Nacional de Descripción: Orientações para a } \\
\text { Descrição Arquivística I. }\end{array}$ \\
\hline \multirow{2}{*}{2007} & $\begin{array}{l}\text { - Creación por Decreto Ley de la Direcção-Geral de Arquivos (DGARQ) como consecuencia de la } \\
\text { fusión del INA/TT y el Centro Portugués de Fotografía. } \\
\text { La DGARQ fue el responsable de continuar con el desarrollo de las orientaciones, es decir, del } \\
\text { resto de partes que componían la norma portuguesa. }\end{array}$ \\
\hline \multirow{2}{*}{2007} & $\begin{array}{l}\text { - Se finaliza la segunda y tercera parte de las Orientações para a Descrição Arquivística II y III. } \\
\text {-Se publica ODA en internet (páginas web de Portugal y en la página oficial del CIA). } \\
\text { archivos regionales y en los archivos municipales del país. }\end{array}$ \\
\hline
\end{tabular}

Fuente: Elaborado por la autora.

Las Orientações para a Descrição Arquivística (ODA) fue redactada en varias etapas y, antes de su publicación definitiva, fue consultada la comunidad archivística. De esta forma, la DGARQ pretendía obtener un instrumento aprobado por todos los profesionales y que lograra un consenso entre las diferentes prácticas archivísticas.

ODA, como su nombre indica, son orientaciones generales y, como tales, no influyen sobre la documentación con características específicas (fotografía, mapas, documentos sonoros, entre otros). No obstante, a través de ODA se pueden obtener descripciones de varias entidades archivísticas: documentos y productores, además se pueden crear puntos de acceso. En la actualidad, los archiveros portugueses trabajan con el fin de alcanzar en sus proyectos descriptivos una articulación entre las diferentes normas y sus orientaciones. Buscan una coherencia entre los modelos conceptuales adoptados y su implementación práctica.

\section{EL PROCESO DE NORMALIZACIÓN EN BRASIL: NOBRADE}

La publicación de $\operatorname{ISAD}(G)$ no representó demasiado para los archiveros brasileños. Sólo se había impreso, a principios de los 90 , la traducción de $\operatorname{ISAD}(G)$ realizada por la Asociación Portuguesa de Bibliotecarios, Archivistas y Documentalistas y tenía una escasa circulación en el país.

En 1996, en el XIII Congreso Internacional de Archivos, se creó un Comité del CIA sobre normas descriptivas, responsable de la revisión, difusión y mantenimiento de las normas generales existentes (BONAL ZAZO, 2000, p. 136). La participación de Brasil en este proceso de revisión demostró la necesidad de traducir la norma internacional. El Archivo Nacional se responsabilizó de esta tarea y en 1998 publica la primera traducción brasileña de ISAD(G) y del borrador de ISAAR(CPF). Esta versión fue distribuida, gratuitamente, en el país con el fin de promoverlas y realizar eventos para discutirlas. 
La presencia de archiveros brasileños en este comité de revisión de la norma internacional fue muy positiva. La comunidad archivística ganó en experiencia y tuvo un contacto amplio y constante con los profesionales de otros países lo que los ayudó a profundizar y reflexionar sobre la normativa del CIA.

Posteriormente, se comenzaron a organizar reuniones con profesionales que participaron en el proceso de formación y traducción de la normativa internacional.

\section{NORMALIZACIÓN DE LA DESCRIPCIÓN ARCHIVÍSTICA EN ESPAÑA.}

España es uno de los países europeos con mayor tradición archivística, sin embargo, no cuenta, aún, con una norma nacional de descripción completa. En este país el interés por la normalización aumenta a finales de la década de los 80 , vinculado al desarrollo de las nuevas tecnologías de la información y al proceso de normalización internacional desarrollado por el CIA.

El proyecto de normalización en España, hay que analizarlo, orientado a dos perspectivas: una a nivel nacional y otra a nivel de comunidades autónomas. En el país ibérico, desde el 2000 y hasta la actualidad, se han venido desarrollando varios proyectos normativos tanto de carácter nacional como regional. De esta forma, el Sistema de Archivos Estatales cuenta con dos normas: NEDA-I (Borrador de la versión 1 de la Norma Española para la Descripción Archivística - primera versión del proyecto) y NEPAN (Norma para la Elaboración de los Puntos de Acceso). Además, algunas comunidades autónomas como Castilla y león, Cataluña, Galicia y Aragón han elaborado sus propios estándares para la descripción de sus entidades archivísticas.

Para comprender mejor el desarrollo normativo en España, a continuación se ejemplificarán los procesos de normalización y las actividades desarrolladas en este país (a nivel nacional y regional) para desarrollar los actuales estándares descriptivas que poseen. 
Tabla 5. Actividades realizadas en España para normalizar la descripción.

\begin{tabular}{|c|c|}
\hline \multicolumn{2}{|r|}{ PROCESO DE NORMALICACIÓN EN ESPAÑA } \\
\hline FECHAS & ACTIVIDADES \\
\hline Década del 90 & $\begin{array}{l}\text {-Trabajos a nivel nacional de regulación de la estructura y contenido de datos de la } \\
\text { descripción archivística a nivel de sistemas específicos de descripción o de determinados } \\
\text { archivos. }\end{array}$ \\
\hline 1999 & $\begin{array}{l}\text { - Creación de un Grupo de Trabajo de Normalización en Castilla y León, formado por tres } \\
\text { profesionales de la archivística de la región, para desarrollar un estándar de descripción } \\
\text { adaptado a las normas internacionales. }\end{array}$ \\
\hline 2000 & $\begin{array}{l}\text { - Presentación en el Congreso Internacional de Archivos de Sevilla de la primera edición del } \\
\text { Manual de Descripción Multinivel (MDM) de Castilla y León. }\end{array}$ \\
\hline 2001 & $\begin{array}{l}\text { - Se crea un grupo de trabajo en la Comunidad Autónoma de Cataluña para dirigir y } \\
\text { elaborar la norma de descripción de esta comunidad. } \\
\text { - Se elabora el Proyecto de Norma de Descripción Archivística de Cataluña (NODAC). }\end{array}$ \\
\hline 2001 & $\begin{array}{l}\text { - Constitución, a nivel nacional, del Grupo de Trabajo de Representantes de la } \\
\text { Administración Central y Administraciones Autonómicas (GTACAA) para la elaboración de } \\
\text { una Norma Española de Descripción Archivística (NEDA). }\end{array}$ \\
\hline 2001 & $\begin{array}{l}\text { - Se crea en la Comunidad Autónoma de Aragón el Grupo de Trabajo de Autoridades de } \\
\text { Aragón para dirigir y elaborar varias normas que después puedieran complementarse. }\end{array}$ \\
\hline 2003 & $\begin{array}{l}\text { - Se crea en la Comunidad Autónoma de Galicia un grupo de trabajo para elaborar un } \\
\text { estándar en esta región. }\end{array}$ \\
\hline 2005 & $\begin{array}{l}\text { - El grupo de trabajo de normalización, a nivel nacional, dirigido por la Subdirección General } \\
\text { de Archivos finaliza NEDA1 (Borrador de la versión } 1 \text { de la Norma Española de Descripción } \\
\text { Archivística). Este borrador sólo desarrolla los seis elementos obligatorios propuesto en } \\
\text { ISAD(G). }\end{array}$ \\
\hline 2007 & - Se publica, oficialmente, la Norma de Descripción Archivística de Cataluña (NODAC). \\
\hline 2007 & $\begin{array}{l}\text { - Publicación de la Orden CUL/1524/2007, de } 25 \text { de mayo, por la que se crea una nueva } \\
\text { comisión nacional para trabajar en la norma español: Comisión de Normas Españolas de } \\
\text { Descripción Archivística (CNEDA). }\end{array}$ \\
\hline 2007 & $\begin{array}{l}\text { - Primera sesión ordinaria de la CNEDA: Aprobación del proyecto NEDAl, en el que se } \\
\text { establecen las líneas esenciales del proyecto normativo a realizar durante } \\
\text { aproximadamente } 5 \text { años. }\end{array}$ \\
\hline 2008 & - Se publica la versión final de la Norma Gallega de Descripción Archivística (NOGADA). \\
\hline 2008 & $\begin{array}{l}\text { - La Subdirección General de Archivos Españoles crea el Grupo de Indización de los Archivos } \\
\text { Estatales con el objeto de crear una norma sobre puntos de acceso de autoridades } \\
\text { archivísticas. }\end{array}$ \\
\hline 2008 & $\begin{array}{l}\text { El grupo de trabajo de Aragón publica ARANOR. Norma Aragonesa para la Descripción de } \\
\text { Autoridades de Archivos. }\end{array}$ \\
\hline 2009 & $\begin{array}{l}\text { Publicación del borrador del Modelo Conceptual de la Norma Española de Descripción } \\
\text { Archivística en la página web de la CNEDA. Este borrador contenía, sólo, la primera parte } \\
\text { referente a los tipos de entidad. }\end{array}$ \\
\hline 2010 & $\begin{array}{l}\text { El grupo de trabajo de Aragón publica la Norma de Estructura de Datos de Archivos } \\
\text { Aragoneses (EDARA). }\end{array}$ \\
\hline 2010 & $\begin{array}{l}\text { El Grupo de Indización de los Archivos Estatales publica la primera la Norma para la } \\
\text { Elaboración de Puntos de Acceso Normalizados de Nombres de Instituciones, Personas, } \\
\text { Familias, Lugares y Materias para el Sistema de Archivos Estatales de España. }\end{array}$ \\
\hline 2012 & $\begin{array}{l}\text { Publicación final del "Modelo conceptual de descripción archivistica y requisitos de datos } \\
\text { básicos de las descripciones de documentos de archivo, agentes y funciones", primera } \\
\text { parte (Tipos de entidad) y segunda parte (Relaciones). Este documento es el resultado } \\
\text { esperado de la actividad } 1 \text { del Proyecto NEDA-I. }\end{array}$ \\
\hline $\begin{array}{c}2012- \\
\text { Actualidad }\end{array}$ & La CNEDA sigue trabajando en el proyecto de NEDA. \\
\hline
\end{tabular}

Fuente: Elaborado por la autora. 
En España se ha trabajado, intensamente, para obtener una normativa nacional que sistematice la descripción en los archivos estatales españoles. Como resultado cuentan con el mencionado borrador de NEDAI y la Norma para la Elaboración de Puntos de Acceso. Durante todo el proceso de normalización de la descripción se ha consultado, siempre, a la comunidad archivística española para obtener sus sugerencias y observaciones críticas. De esta forma, la Subdirección General de Archivos Estatales pretende obtener un estándar que cuente con la aceptación de todos los profesionales del área y un consenso general entre las diferentes prácticas y tendencias archivísticas del conjunto de comunidades autónomas, tarea complicada en el país ibérico.

En la actualidad, la Comisión de Normas Españolas de Descripción Archivística se encuentra trabajando, intensamente, en el desarrollo de las Normas Españolas para la Descripción Archivística. España es de los pocos países que ha desarrollado una base conceptual sólida a partir de la cual se elaborarán las pautas para la descripción de los diferentes tipos de entidades archivísticas y sus relaciones. Este modelo conceptual ha tenido un impacto y un gran reconocimiento por parte de la comunidad archivística nacional e internacional y así se ha manifestado en diferentes reuniones y eventos dentro y fuera de España.

Por su parte, el MDM de Castilla y León es el resultado más sobresaliente alcanzado por esta comunidad en materia de descripción archivística. El éxito de la primera edición del manual fue tal que en el 2001 se efectuó una segunda impresión del estándar y una segunda edición en el año 2006. En la actualidad este texto normativo es utilizado por varios archivos de toda España y de otros países del ámbito Iberoamericano. Además, constituye fuente indispensable de consulta para la propuesta de nuevos proyectos normativos nacionales.

En tanto, la comunidad de Cataluña ya cuenta con NODAC; norma creada para desarrollar ISAD(G) y hacerla compatible con otras normas específicas y adecuarla a la realidad de los archivos catalanes. Con la publicación de NODAC no termina el proceso de normalización de la descripción archivística en Cataluña. Los archiveros catalanes dejan abierto el capítulo de registros de autoridades, de regulaciones para la creación de puntos de acceso y de formatos de intercambio de información en ambientes electrónicos.

La comunidad autónoma de Galicia elabora y publica NOGADA. Este proyecto normativo facilita la aplicación de la norma internacional en los archivos gallegos e integra a sus archiveros en los movimientos internacionales y nacionales de normalización de la descripción. Según el grupo que trabajó en este estándar, NOGADA es una respuesta a los problemas profesionales en materia de descripción y articula un nivel de consenso primario 
que opera por debajo y en estrecha relación con otros niveles más amplios de ámbito estatal o internacional (PEREIRA OLIVEIRA, 2008).

Por último, no se puede dejar de mencionar Aragón. En esta comunidad autónoma se desarrolló la Norma Aragonesa para la descripción de Autoridades de Archivos. Este estándar, elaborado por el Grupo de Trabajo de Autoridades de Aragón, pretende servir como guía para la elaboración de registros de autoridad de instituciones, personas y familia siguiendo las directrices y la estructura que ofrece ISAAR(CPF). Un resultado concreto de normalización en esta región lo constituye, también, EDARA: la Estructura de Datos de Archivos Aragoneses.

Tal y como se puede observar, son varios los resultados obtenidos en la normalización de la descripción archivística en España y algunas de sus comunidades autónomas. Todos estos proyectos son válidos $\mathrm{y}$, sin lugar a dudas, han aportando experiencias prácticas y teóricas válidas para muchos países.

\section{RESULTADOS}

Mediante este estudio ha sido posible obtener una visión general de los modelos de trabajo utilizados para la confección de estas normas nacionales y regionales. El análisis de estos procesos permite conocer una serie de aspectos de gran utilidad para todos aquellos países y regiones interesados en la elaboración sus propias normas de descripción.

Han sido identificados los elementos más representativos; aunque se entiende que un estudio más profundo sobre el proceso de elaboración de normas desvelará, posiblemente, otros aspectos pero serán de menor interés. A continuación se listarán cuáles son las actividades o directrices que se tuvieron en cuenta en estos países para elaborar los estándares citados.

8.1 Nombramiento de una institución de carácter nacional o autónomo para dirigir el proyecto de normalización de la descripción archivística.

Tabla 6. Instituciones responsables de la creación de las normas de descripción.

\begin{tabular}{|l|l|l|}
\hline \multicolumn{1}{|c|}{ LUGARES } & \multicolumn{1}{|c|}{ NORMAS } & \multicolumn{1}{c|}{ DIRECCIÓN DEL PROYECTO } \\
\hline EE.UU & DACS & Society of American Archivists. \\
\hline CANADÁ & RAD2 & $\begin{array}{l}\text { Bureau of Canadian Archivists. } \\
\text { Canadian Council of Archives. } \\
\text { National Archives of Canada. }\end{array}$ \\
\hline $\begin{array}{l}\text { GRAN } \\
\text { BRETAÑA }\end{array}$ & RCNPLI & National Council of Archives. \\
\hline PORTUGAL & ODA & $\begin{array}{l}\text { Instituto dos Arquivos Nacionais /Torre do Tombo. } \\
\text { Direção-Geral de Arquivos. }\end{array}$ \\
\hline BRASIL & NOBRADE & Conselho Nacional de Arquivos. \\
\hline
\end{tabular}




\begin{tabular}{|l|l|l|}
\hline ESPAÑA & $\begin{array}{l}\text { NEDA } \\
\text { NEPAN }\end{array}$ & $\begin{array}{l}\text { Ministerio de Cultura de España. Dirección General } \\
\text { del Libro, Archivos y Bibliotecas. Subdirección } \\
\text { General de los Archivos Estatales. }\end{array}$ \\
\hline $\begin{array}{l}\text { CASTILLA Y } \\
\text { LEÓN }\end{array}$ & MDM & $\begin{array}{l}\text { Junta de Castilla y león. Consejería de Educación y } \\
\text { Cultura. Dirección General de Patrimonio y } \\
\text { Promoción Cultural. }\end{array}$ \\
\hline CATALUÑA & NODAC & $\begin{array}{l}\text { Generalitat de Catalunya. Departament de Cultura i } \\
\text { Mitjans de Comunicació. Server d Arxius. }\end{array}$ \\
\hline GALICIA & NOGADA & $\begin{array}{l}\text { Junta de Galicia. Subdirección General del } \\
\text { Patrimonio Cultural. Servicio de Archivos y Museos. }\end{array}$ \\
\hline
\end{tabular}

Fuente: Elaborado por la autora.

Como se puede observar, en todos los procesos analizados, los órganos rectores de los sistemas de archivos nacionales o regionales fueron los responsables de dirigir, supervisar y apoyar la realización de las normas de descripción archivística. Con excepción de EE.UU. donde el proceso fue controlado por una asociación profesional: Society of American Archivists. La dirección de una institución rectora facilitó un mejor desempeño del trabajo pues estas instituciones patrocinaron los proyectos y los impulsaron en todo lo relacionado con cuestiones financieras, políticas y prácticas. Además, una vez finalizados los proyectos, los órganos directivos tuvieron la autoridad de convertir los proyectos en estándares oficiales para que pudieran ser utilizados en todos los tipos de archivos del país o de la región.

8.2. Formación de un comité o grupo de normalización para la creación de la norma de descripción archivistica.

La creación de un comité o grupo de trabajo responsable de la elaboración de la norma es una de las actividades fundamentales para la elaboración de cualquier estándar. En todos los casos analizados esta fue la primera acción que se realizó. La formación de un equipo integrado por especialistas con experiencias en la normalización y en la práctica de la descripción fueron factores clave del éxito normativo. En todos los países los grupos fueron los coordinadores del proyecto y los responsables de la redacción, revisión, edición y publicación de la norma.

Tabla 7. Comité para la creación de la norma de descripción archivística.

\begin{tabular}{|l|l|l|}
\hline \multicolumn{1}{|c|}{ LUGARES } & \multicolumn{1}{|c|}{ NORMAS } & \multicolumn{1}{c|}{ COMITÉ DE NORMALIZACIÓN } \\
\hline EE.UU. & DACS & Technical Subcomité for Descriptive Standards \\
\hline CANADÁ & RAD2 & Canadian Committee an Archival Committee \\
\hline $\begin{array}{l}\text { GRAN } \\
\text { BRETAÑA }\end{array}$ & RCNPLI & $\begin{array}{l}\text { Comité de Redacción del Fichero Nacional de } \\
\text { Autoridades }\end{array}$ \\
\hline PORTUGAL & ODA & $\begin{array}{l}\text { Grupo de Trabalho de Normalização da Descrição } \\
\text { em Arquivo }\end{array}$ \\
\hline BRASIL & NOBRADE & $\begin{array}{l}\text { Câmara Técnica de Normalização da Descrição } \\
\text { Arquivística }\end{array}$ \\
\hline
\end{tabular}




\begin{tabular}{|l|l|l|}
\hline ESPAÑA & $\begin{array}{l}\text { NEDA } \\
\text { NEPAN }\end{array}$ & $\begin{array}{l}\text { Comisión Nacional de Descripción Archivística } \\
\text { Grupo de Indización }\end{array}$ \\
\hline $\begin{array}{l}\text { CASTILLA Y } \\
\text { LEÓN }\end{array}$ & MDM & $\begin{array}{l}\text { Grupo de Trabajo para la Normalización de la } \\
\text { Descripción }\end{array}$ \\
\hline CATALUÑA & NODAC & $\begin{array}{l}\text { Grupo de Trabajo para la Normalización de la } \\
\text { Descripción }\end{array}$ \\
\hline GALICIA & NOGADA & Grupo de Trabajo de Archiveros de Galicia \\
\hline
\end{tabular}

Fuente: Elaborado por la autora.

8.3 Selección de especialistas de diversas instituciones y asociaciones vinculadas a las ciencias de la información para formar los comités de normalización.

En todos los procesos normativos analizados se seleccionaron profesionales de instituciones relacionadas con la archivística, la biblioteconomía y la ciencia de la información en general. En mayor número están representados los archivos, seguidos por las universidades y las bibliotecas; en menor medida, aparecen los museos y otras instituciones vinculadas al mundo de la información. En el siguiente gráfico, realizado a partir de los datos obtenidos en esta investigación, se muestran las tendencias seguidas en la composición de los comités de normalización.

Figura 1. Composición de los comités de normalización en los países y comunidades estudiadas.

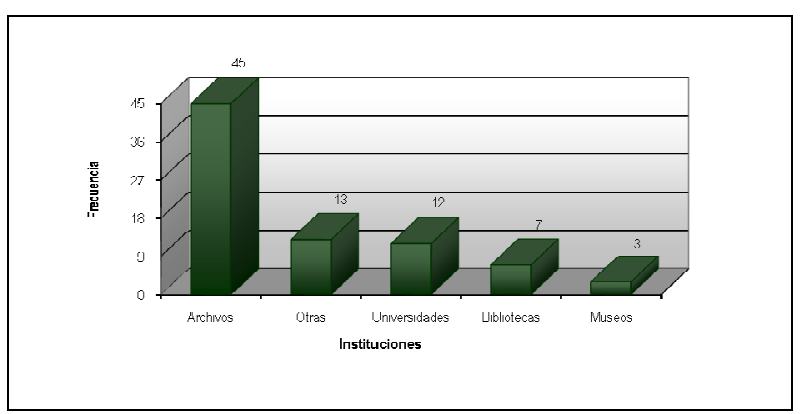

Fuente: Elaborado por la autora.

Resulta interesante conocer cuáles fueron las tendencias seguidas en los países anglosajones, los de habla portugués y en España para constituir los comités de normalización. En los tres gráficos siguientes se ejemplifican por grupos de países la cantidad de instituciones que participaron. 
Figura 2. Composición de los comités de normalización por grupo de países.
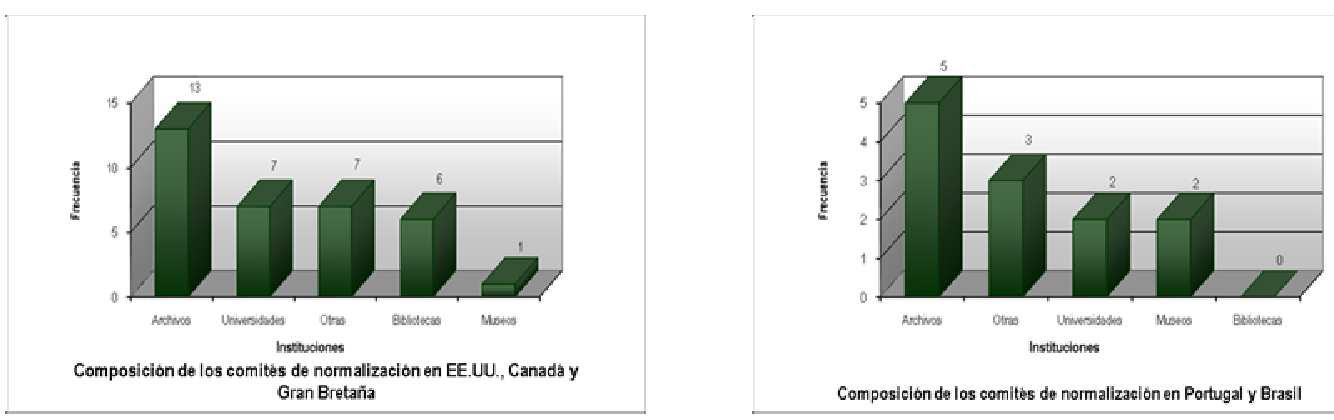

Composición de los comitès de normalización en Portugal y Brasil

Fuente: Elaborado por la autora.

Como se puede apreciar en las figuras anteriores, los equipos de trabajo de los países anglosajones estuvieron formados por profesionales de archivos, bibliotecas, museos, universidades y otras instituciones, con mayor representatividad de los archivos y las bibliotecas. La presencia de un mayor número de profesionales del ámbito de las bibliotecas en estos países explica la gran influencia que han tenido del campo de la biblioteconomía los estándares anglosajones.

En los países de habla portuguesa los grupos estuvieron formados por profesionales de archivos, museos, universidades y otras instituciones, dejando fuera especialistas del ámbito de las bibliotecas, en estos casos la mayor representatividad correspondió a los archivos seguidos de las universidades. De ahí la tendencia fuertemente archivística de los dos estándares.

En el caso de España y las comunidades autónomas, que han desarrollado normas de descripción, los grupos han estado integrados por profesionales, en su mayoría, de los archivos. También formaron parte de las comisiones algunas universidades y otros centros, vinculado a las instituciones archivísticas. En España no se han incluido en los grupos 
profesionales del ámbito de la biblioteconomía, de ahí la escasa influencia en los estándares de esta área del saber.

Se puede afirmar que para el éxito de una norma de descripción archivística, se necesita contar con la experiencia y los conocimientos de todos los especialistas vinculados con las ciencias de la información, dígase archiveros, bibliotecarios, museólogos, profesores, informáticos, lingüistas y demás profesionales vinculados al área.

8.4 Actividades desarrolladas por los comités de normalización para la creación de las normas.

En el marco operativo de los grupos de normalización se desarrollaron un conjunto de actividades cuyo alcance se señala seguidamente:

- Formación de grupos de trabajos para atender temas específicos.

- Redacción de un programa de trabajo con las actividades y objetivos a desarrollar.

- Realización de un estudio teórico y práctico de la normalización de la descripción archivística en cada país.

- Intercambio con la comunidad archivística para la construcción de la norma.

- Redacción de borradores antes de la publicación oficial de la norma.

Para la redacción de las normas RCPPCN, RAD2, NOGADA y NEPAN se crearon grupos con el fin de trabajar en temas específicos. En el caso de RAD2 y NOGADA el objetivo de estos grupos fue el trabajo con documentos especiales (audiovisuales, gráficos, electrónicos y filatélicos, entre otros). En el caso de RCPPCN y NEPAN los grupos se formaron para trabajar en la creación de los puntos de acceso de nombres de instituciones, personas, familias, materias y lugares geográficos. En el resto de los estándares no se establecieron grupos independientes para su desarrollo.

Respecto a los programas de trabajo, sólo en el caso de Portugal y Cataluña se contó con un documento que sirvió de guía y calendario para la ejecución del proyecto. Estos programas contaban con los objetivos y actividades que debía seguir el proceso de normalización y los tiempos de desarrollo de las etapas. Hay que destacar que la CNEDA dispone, en la actualidad, con un programa de trabajo bien definido para el desarrollo de cada una de las actividades de la norma española.

En la actividad relacionada con el estudio de la normalización de la descripción archivística, se puede decir que en todos los procesos normativos antes de comenzar la redacción de la norma se realizaron estudios del tema. Se pudo constatar que se analizaron 
aspectos referentes a las normas internacionales, a las normas APPM, RAD y MAD y, también, se revisaron los principales conceptos y principios teóricos de la descripción archivística.

En lo referente al intercambio con la comunidad archivística, en todos los casos se involucró en el proceso de elaboración de la norma a los profesionales vinculados con el tema, con la finalidad de lograr un consenso nacional o regional de las prácticas archivísticas existentes y poder aplicarla en todos los archivos.

En todos los países y regiones, antes de la publicación final de la norma, se realizaron varias versiones de borradores, con el objeto de eliminar posibles errores teóricos - prácticos y poder, con ello, obtener una edición oficial corregida y revisada.

Es importante analizar este conjunto de actividades antes de comenzar el desarrollo de una normativa nacional.

8.5 Tiempo empleado en la elaboración de los estándares.

Resulta de interés exponer los datos relacionados con la duración del proceso de desarrollo de las normas desde la constitución de los grupos hasta la edición de los estándares. Esto permite conocer el tiempo estimado para desarrollar este tipo de proyectos. A continuación se expone una tabla que recoge estas fechas.

Tabla 8. Período de tiempo empleado en la elaboración de los estándares.

\begin{tabular}{|c|c|c|c|c|}
\hline \multirow{2}{*}{ LUGARES } & \multirow{2}{*}{ NORMAS } & \multicolumn{2}{|c|}{$\begin{array}{l}\text { PERÍODO DE FORMACIÓN } \\
\text { DE LOS ESTÁNDARES }\end{array}$} & \multirow{2}{*}{$\begin{array}{c}\text { TOTAL } \\
\text { DE } \\
\text { AÑ̃S }\end{array}$} \\
\hline & & $\begin{array}{l}\text { FECHA DE } \\
\text { INICIO }\end{array}$ & $\begin{array}{l}\text { FECHA DE } \\
\text { FIN }\end{array}$ & \\
\hline $\begin{array}{l}\text { GRAN } \\
\text { BRETAÑA }\end{array}$ & RCPPCN & 1990 & 1997 & 7 \\
\hline $\begin{array}{l}\text { GRAN } \\
\text { BRETAÑA }\end{array}$ & MAD3 & 1998 & 2000 & 2 \\
\hline EE.UU. & DACS & 1996 & 2004 & 8 \\
\hline CANADÁ & RAD2 & 1996 & 2008 & 12 \\
\hline PORTUGAL & ODA & 1996 & 2007 & 11 \\
\hline BRASIL & NOBRADE & 2001 & 2007 & 6 \\
\hline ESPAÑA & NEPAN & 2008 & 2010 & 2 \\
\hline $\begin{array}{l}\text { CASTILLA Y } \\
\text { LEÓN }\end{array}$ & MDM & 1999 & 2000 & 1 \\
\hline CATALUÑA & NODAC & 2001 & 2007 & 6 \\
\hline GALICIA & NOGADA & 2003 & 2008 & 5 \\
\hline
\end{tabular}

Fuente: Elaborado por la autora.

Los años empleados para la formación de los estándares varía en cada país y la extensión de los períodos depende de diversas razones. En MAD3, MDM y NEPAN el tiempo 
empleado para su elaboración y publicación definitiva fue muy corto, uno y dos años aproximadamente. Hay que destacar que los manuales fueron elaborados por autores independientes, aspecto que facilita el desarrollo del trabajo; el texto británico, además, se realizó sobre la base de su primera versión. En EE.UU y Canadá los comités de normalización emplearon un período de tiempo más extenso porque tuvieron como antecedente el fracaso del proyecto común CUSTARD, factor que retraso la edición de las dos normas nacionales de forma independiente. Portugal realizó sus orientaciones en cuatro fases, de ahí el amplio período utilizado hasta la publicación definitiva de ODA. Para el resto de las normas el tiempo utilizado se sitúa entre los 5 y 7 años. Los nuevos proyectos demoran menos años en la proyección y edición de las normas descriptivas, pues se apoyan en las experiencias en materia de normalización desarrolladas por otros sistemas de archivos.

\section{CONSIDERACIONES FINALES}

Como se puede observar en lo anteriormente descrito, seis países ya han desarrollado y publicado sus propias normas nacionales, además de los exitosos proyectos normativos que han desarrollado varias comunidades autónomas de España. En un período de casi 20 años la comunidad archivística internacional cuenta con un gran número de normas de estructuras de datos, contenidos, presentación y formatos de codificación. Todos estos estándares son consecuencia del esfuerzo de un determinado grupo de profesionales que trabajaron en la adaptación de las normas internacionales a la realidad archivística de cada país o región.

Figura 3. Evolución histórica de las normas de descripción.

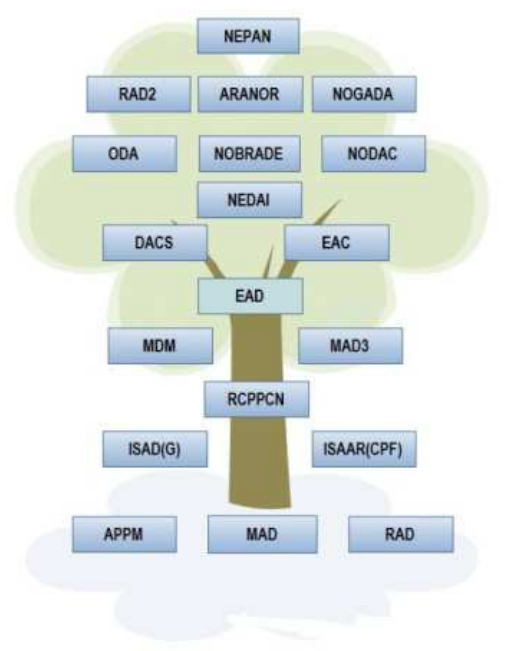

Fuente: Elaborado por la autora. 
En la figura anterior se ejemplifica la evolución histórica de los estándares descriptivos. Las normas publicadas por el CIA se basaron en las normas existentes en EE.UU, Canadá y Gran Bretaña, es decir, tomaron como base las Archives, Personal Papers and Manuscripts, el Manual of Archival Description y las Rules Archival Description para elaborar sus propuestas internacionales. A partir de la edición de ISAD(G) e ISAAR(CPF) se revolucionó la regulación de las descripciones y el resultado final ha sido la elaboración y la publicación de los estándares que se han mencionado.

Gran Bretaña fue de los primeros países en desarrollar estándares de datos y contenidos para describir entidades archivísticas. Posteriormente, EE.UU y Canadá desarrollaron esfuerzos conjuntos para elaborar una norma norteamericana descripción; después del fracaso de este proyecto común cada uno de ellos publicó sus propias normativas descriptivas, basadas en APPM, RAD y CUSTARD. En España una comunidad autónoma fue la primera en elaborar un manual descriptivo (Castilla y León) y en el año 2005 una comisión nacional elabora lo que se conoce como el primer borrador de la Norma Española de Descripción Archivística. En el año 2007, se publican, oficialmente, varios textos normativos en diferentes países y comunidades autonómicas: Portugal, Brasil y la Comunidad Autónoma de

21 Cataluña. En España se siguen proyectado y publicando estándares, ejemplo de ellos son: NOGADA, ARANOR y, recientemente, EDARA y NEPAN.

La publicación de este conjunto de instrumentos descriptivos ha supuesto un punto final a las extensas discusiones y reflexiones en el ámbito de los archivos relacionadas con la objeción a normalizar cualquier tipo de proceso, en especial el de descripción. Los archiveros han pasado de contar con una escasa lista de normas para la actividad de descripción a un listado interminable de estándares que brindan soluciones para disímiles casos presentados a diario en el quehacer de las instituciones. Todas estas normas están abiertas al cambio y a posibles revisiones orientadas a su mejora, y es posible, por tanto, afirmar que todas han conseguido incrementar la accesibilidad y el uso de los documentos archivístico.

\section{Referencias Bibliográficas}

ARANOR. NORMA ARAGONESA PARA LA DESCRIPCIÓN DE AUTORIDADES DE ARCHIVOS. Aragón: Gobierno de Aragón, Departamento de Educación Cultura y Deporte, 2008. Disponible en: <http://www.anabad.org/admin/archivo/docdow.php?id=506>. Acceso en: 05 de abr. 2014.

BONAL ZAZO, J.L. La descripción archivística normalizada: origen, fundamentos, principios y técnicas. Gijón: Trea, 2000. 
COOK, M.; PROCTER, M. Manual of Archival description. 2.ed. Aldershot: Gower, 1989.

COOK, M.; PROCTER, M. Manual of Archival Description. 3. ed. Aldershot: Gower, 2000.

COMISIÓN DE NORMAS ESPAÑOLAS DE DESCRIPCIÓN ARCHIVÍSTICA. Modelo conceptual de descripción archivística y requisitos de datos básicos de las descripciones de documentos de archivo, agentes y funciones. Parte 1: tipos de entidad. Parte 2: Relaciones. Madrid: CNEDA, 2012. Disponible en: < http://www.mcu.es/archivos/docs/NEDA_MCDA_P1_P2_20120618.pdf >. Acceso en: 06 feb. 2014.

CONSEJO INTERNACIONAL DE ARCHIVOS. Declaración de Principios sobre la Descripción Archivística: Adoptada por la Comisión Ad Hoc sobre Normas de Descripción. Madrid, 1992. Ottawa: Secretaria de la Comisión sobre Normas de Descripción, 1992.

.ISAAR (CPF): Norma Internacional sobre los Registros de Autoridad de Archivos Relativos a Instituciones, Personas y Familias. Adoptada por el Comité de Normas de Descripción. 2. ed. Madrid: Dirección General del Libro, Archivos y Bibliotecas, Subdirección General de los Archivos Estatales, 2004.

ISAD (G): Norma internacional para la descripción archivística. Adoptada por la Comisión Ad Hoc sobre Normas de Descripción, Estocolmo, Suecia, 19-22 de septiembre de 1999. Madrid: Dirección General del Libro, Archivos y Bibliotecas, 2000.

DESCRIBING ARCHIVES: A CONTENT STANDARD. Chicago: Society of American Archivists, 2007.

DEVELOPMENT OF THE ENCODED ARCHIVAL DESCRIPTION. Disponible en: <http://www.loc.gov/ead/eaddev.html>. Acceso en: 05 de abr. 2014.

DUFF, W. M. Normas de descripción archivística. Tabula, n.11, p. 51-64, 2008.

EAC-CPF: ENCODED ARCHIVAL CONTEXT - CORPORATE BODIES, PERSONS, AND FAMILIES, 2004. Disponible en: <http://eac.staatsbibliothek-berlin.de/>. Acceso en: 07 de abr. 2014.

EDARA: ESTRUCTURA DE DATOS DE LOS ARCHIVOS ARAGONESES, 2010. Disponible en: $<$ http://www.dehuesca.es/ sipca/IMAGEN/documentos_web/Norma_edara_v1.0_caratula.p df>. Acceso en: 06 de abr. 2014.

FONSECA, V. M. M. A Normalização da Descrição Arquivística - avanços internacionais e a situação do Brasil, $2001 . \quad$ Disponible en: $<$ http://www.conarq.arquivonacional.gov.br/Media/publicacoes/mesa/a normalizao da desc rio arquivstica avanos.pdf >. Acceso en: 03 de abr. 2014.

HERNÁNDEZ MARTíN, A.; HERNÁNDEZ SÁNCHEZ, M. Proyecto CUSTARD: La iniciativa americana para la descripción. Archivamos, n. 47-48, p. 22-28, 2003.

MANUAL DE DESCRIPCIÓN MULTINIVEL: PROPUESTA DE ADAPTACIÓN DE LAS NORMAS INTERNACIONALES DE DESCRIPCIÓN ARCHIVÍSTICA. Salamanca: Junta de Castilla y León, 2000. NOBRADE: NORMA BRASILEIRA DE DESCRIÇÃO ARQUIVÍSTICA. Río de Janeiro: Consejo Nacional de Arquivos, 2007.

NORMA DE DESCRIPCIÓN ARCHIVÍSTICA DE CATALUÑA: NODAC. Barcelona: Generalitat de Cataluña, Departamento Cultura y Medios de Comunicación, 2007. Disponible en: 
<http://www.aefp.org.es/NS/Documentos/NormasDescriptivas/NODACesp.pdf>. Acceso en: 02 de abr. 2014.

NORMA ESPAÑOLA DE DESCRIPCIÓN ARCHIVÍSTICA (NEDA).1.ed. Madrid: Ministerio de Cultura, Subdirección General de Archivos Estatales, 2005.

NORMA GALEGA DE DESCRIPCIÓN ARQUIVÍSTICA (NOGADA). PROPOSTA INICIAL. [s.I.]: Grupo de Arquiveiros de Galicia, 2008.

NORMA PARA LA ELABORACIÓN DE PUNTOS DE ACCESO NORMALIZADOS DE NOMBRES DE INSTITUCIONES, PERSONAS, FAMILIAS, LUGARES Y MATERIAS EN EL SISTEMA DE DESCRIPCIÓN ARCHIVÍSTICA DE LOS ARCHIVOS ESTATALES. Madrid: Ministerio de Cultura, Subdirección General de Archivos Estatales, 2010. Disponible en: <http://www.mcu.es/archivos/Novedades/novedades_Puntos_Acceso_Normalizados.html>. Acceso en: 31 de mar. 2014.

ORIENTAÇÕES PARA A DESCRIÇÃO ARQUIVÍSTICA. 2. ed. Lisboa: DGARQ, 2007.

PEREIRA OLIVEIRA, M. D. La Norma Gallega de Descripción Archivística. Criterios de elaboración, estructura y contenido. En: CONGRESO DE ARCHIVOS DE CASTILLA Y LEÓN, 4, 2008, León. España. Disponible en: <http://www.acal.es/Portals/4/congreso leon/Grupo trabajo archiveros Galicia.pdf>. Acceso en: 31 de mar. 2014.

RULES FOR ARCHIVAL DESCRIPTION. Ottawa: Bureau Canadien des Archivistes, 2008. Disponible en: < http://www.cdncouncilarchives.ca/archdesrules.html>. Acceso en: 01 de abr. 2014.

RULES FOR THE CONSTRUCTION OF PERSONAL, PLACE AND CORPORATE NAMES. UK: National Council on Archives, $1997 . \quad$ Disponible en: <https://www.nationalarchives.gov.uk/documents/information-management/namingrules.pdf>. Acceso en: 30 de mar. 2014. 